University of South Carolina

Scholar Commons

$1-15-2004$

\title{
Energy Conserving Approximations to the Quantum Potential: Dynamics with Linearized Quantum Force
}

\author{
Sophya Garashchuk \\ University of South Carolina--Columbia, sgarashc@chem.sc.edu \\ Vitaly A. Rassolov \\ University of South Carolina - Columbia, rassolov@chem.sc.edu
}

Follow this and additional works at: https://scholarcommons.sc.edu/chem_facpub

Part of the Chemistry Commons

\section{Publication Info \\ Published in Journal of Chemical Physics, Volume 120, Issue 3, 2004, pages 1181-. \\ (c) Journal of Chemical Physics 2004, American Institute of Physics.}

This Article is brought to you by the Chemistry and Biochemistry, Department of at Scholar Commons. It has been accepted for inclusion in Faculty Publications by an authorized administrator of Scholar Commons. For more information, please contact digres@mailbox.sc.edu. 


\title{
Energy conserving approximations to the quantum potential: Dynamics with linearized quantum force
}

\author{
Sophya Garashchuk ${ }^{\text {a) }}$ and Vitaly A. Rassolov \\ Department of Chemistry \& Biochemistry, University of South Carolina, Columbia, South Carolina 29208
}

(Received 29 August 2003; accepted 21 October 2003)

\begin{abstract}
Solution of the Schrödinger equation within the de Broglie-Bohm formulation is based on propagation of trajectories in the presence of a nonlocal quantum potential. We present a new strategy for defining approximate quantum potentials within a restricted trial function by performing the optimal fit to the log-derivatives of the wave function density. This procedure results in the energy-conserving dynamics for a closed system. For one particular form of the trial function leading to the linear quantum force, the optimization problem is solved analytically in terms of the first and second moments of the weighted trajectory distribution. This approach gives exact time-evolution of a correlated Gaussian wave function in a locally quadratic potential. The method is computationally cheap in many dimensions, conserves total energy and satisfies the criterion on the average quantum force. Expectation values are readily found by summing over trajectory weights. Efficient extraction of the phase-dependent quantities is discussed. We illustrate the efficiency and accuracy of the linear quantum force approximation by examining a one-dimensional scattering problem and by computing the wavepacket reaction probability for the hydrogen exchange reaction and the photodissociation spectrum of ICN in two dimensions. () 2004 American Institute of Physics. [DOI: 10.1063/1.1633263]
\end{abstract}

\section{INTRODUCTION}

Quantum-mechanical effects are essential for understanding of a multitude of physical and chemical phenomena, such as zero-point energy, tunneling, interference and nonadiabatic behavior. In particular, time-dependent quantum wavepacket techniques are widely used to study molecular phenomena. Traditional exact methods of solving the timedependent Schrödinger equation that are based on spatial grids, basis sets of functions or discrete variable representation ${ }^{1}$ scale exponentially with the dimensionality $N$ of a system. This scaling makes them inapplicable to systems beyond eight or so dimensions. In contrast, methods of molecular dynamics for which a phase space ensemble of classical trajectories represents a density distribution, are routinely used in studies of large systems of thousands of particles, such as liquids and biomolecules, though these methods are not capable of describing quantum effects. Therefore, intensive research efforts go into development of new approaches incorporating leading quantum effects into trajectory-based methods.

The most popular techniques of this kind are the semiclassical initial value representation methods ${ }^{2-4}$ based on the stationary phase approximation $(\hbar \rightarrow 0)$ to the Schrödinger equation. Trajectories sampling the phase space of an initial wavepacket evolve in time according to classical mechanics; quantum effects are derived from the classical action and from the stability of the final phase space variables with respect to their initial values. The semiclassical initial value representation methods are very appealing in the context of nuclear motion, because the semiclassical limit is appropriate

${ }^{\text {a)} E l e c t r o n i c ~ m a i l: ~ s g a r a s h c @ m a i l . c h e m . s c . e d u ~}$ for systems of large mass and because trajectories can be propagated independently of each other. These methods performed well in various model problems in reactive scattering, nonadiabatic dynamics, dynamics in clusters and spectroscopic applications combined with the filter-diagonalization techniques. ${ }^{4-9}$ Remarkably, recent applications extend to biological systems that are well beyond the full quantum-mechanical theoretical studies due to their size. ${ }^{10,11}$ For example, in Ref. 12 intramolecular proton transfer in photoexcited 2-(2'-hydroxyphenyl)-oxazole was modeled in full-dimension of 35 degrees of freedom. These calculations demonstrated importance of coupling of the reaction modes to the vibrational modes and predicted absorption spectrum. The drawbacks of the semiclassical initial value representation methods are the expensive stability analysis that scales as $N^{2}$, the notorious sign problem of the oscillatory phase space integrals resulting in a very large number of trajectories, ${ }^{12-14}$ and also difficulties in assessing the semiclassical error and in systematically improving semiclassical description toward full quantum mechanics.

A new area of active research deals with approaches based on quantum trajectories that solve the hydrodynamic or de Broglie-Bohm form of the Schrödinger equation. ${ }^{15}$ The wave function of a system is represented in a set of "particles" that move according to the classical equation of motion and carry along certain density. The nonlocal quantum-mechanical character enters this formulation through the quantum potential, which depends on the wave function density and its derivatives. The quantum potential governs the dynamics of the particles along with the classical external potential. Since solution of the Schrödinger equation is based on trajectories, rather than grid points, the scaling 
bottleneck is avoided. Unlike semiclassical initial value representation methods, quantum trajectories sample the coordinate space. In the last few years several practical ways of using quantum trajectories have been suggested, such as local least-square fit, adaptive and moving grids; ${ }^{16-19}$ a methodology of using quantum trajectories within the Wigner representation and in dissipative and nonadiabatic dynamics have been also developed. ${ }^{20-22}$ Application to multidimensional model problems performed with a small number of trajectories is encouraging. ${ }^{23}$ However, for general problems accurate implementation of the hydrodynamic Schrödinger equation, which is a nonlinear equation resulting in a complicated and rapidly varying quantum potential, is challenging. Several intricate examples of quantum trajectories can be found in Refs. 24 and 25, where they serve as an interpretive tool.

Recently, we have introduced the idea of using more practical approximate quantum potentials $(\mathbf{A Q P})^{26,27}$ that can provide accurate description of quantum effects in semiclassical systems. With AQP derived from the global fit to the density we could balance numerical effort vs level of description of quantum effects by choosing the number of fitting Gaussian functions. Complete basis of fitting functions gives exact quantum potential and full quantum-mechanical description, while zero functions gives a "classical" limit. An intermediate number of functions can be considered as a semiclassical description with a single fitting function reproducing exact quantum dynamics of a Gaussian wavepacket in a locally quadratic potential. In principle, the accuracy of the fit controls the degree in which semiclassical description approaches exact quantum dynamics. This was illustrated on a one-dimensional scattering on a barrier for which accurate transmission probabilities were obtained. Nevertheless, the total energy of the system was not conserved. In this paper we describe a different approach to define an approximate quantum potential. This new method of the linearized quantum force (LQF), first described in Ref. 28, is based on the optimal fit within a trial function to the log-derivatives of the wave function density and it conserves total energy in a closed system. We interpret the fitted function as a "nonclassical" component of the momentum operator. A simple implementation with a linear trial function described below is rigorous, free of empirical parameters, and cheap. Optimal parameters of the trial function are found from the first and second moments of distribution of trajectories, whose weights are constant in time. The procedure produces a linear quantum force and it is formulated solely in terms of summation over trajectories. This new method provides exact quantum-mechanical evolution of a Gaussian in a quadratic potential, as do the most successful semiclassical methods, and it is capable of describing quantum mechanical effects for general semiclassical systems. As an illustration we obtained the photodissociation spectra of ICN and the probability of the wavepacket transmission for $\mathrm{H}_{3}$ and its isotopic substitutions in two dimensions.

A multidimensional derivation of the energy conserving approximations to the quantum potential, details of the linearized quantum force approximation and strategies for the wavepacket analysis from weighted trajectory distributions are described in Sec. II. One- and two-dimensional examples are presented in Sec. III along with the analysis of the LQF method. Section IV concludes.

\section{THE ENERGY CONSERVING APPROXIMATE QUANTUM POTENTIAL}

\section{A. The quantum trajectory formalism}

First of all, we briefly describe the quantum trajectory formalism in many dimensions emphasizing the most general features of the quantities involved. Let us consider a quantum mechanical system described in $N$-dimensional Cartesian coordinates with the Hamiltonian $\hat{H}=\hat{P}^{\dagger} M^{-1} \hat{P} / 2+V$, where $M$ is a diagonal matrix of masses, $\left\{M_{n n}\right\}=m^{(n)}{ }^{33}$ Substituting expressions

$$
\psi(\vec{x}, t)=A(\vec{x}, t) \exp \left(\frac{\iota}{\hbar} S(\vec{x}, t)\right), \quad A(\vec{x}, t)=\sqrt{\rho(\vec{x}, t)},
$$

where amplitude $A(\vec{x})$ and phase $S(\vec{x})$ are real functions, into the time-dependent Schrödinger equation and making a transformation into the Lagrangian frame of reference, one obtains

$$
\begin{aligned}
& \frac{d S(\vec{x}, t)}{d t}=\frac{\vec{p}^{T} M^{-1} \vec{p}}{2}-V-U, \\
& \frac{d \rho(\vec{x}, t)}{d t}=-\vec{\nabla} \cdot \vec{v} \rho(\vec{x}, t) .
\end{aligned}
$$

$U$ is a nonlocal time-dependent quantum potential

$$
U=-\frac{\hbar^{2}}{2} \frac{\vec{\nabla}^{T} M^{-1} \vec{\nabla} A(\vec{x}, t)}{A(\vec{x}, t)} .
$$

$\vec{p}=M \vec{v}=\vec{\nabla} S(\vec{x}, t)$ is a classical momentum. Equation (2) is the Hamilton-Jacobi equation describing dynamics of a trajectory in the presence of a classical potential $V$ and a quantum potential $U$. Position and momentum of a trajectory, $\vec{x}$ $=\vec{x}(t)$ and $\vec{p}=\vec{p}(t)$, can be found from Hamilton's equations of motion.

The difference between the action of a quantum mechanical momentum operator, $\hat{P}$, on a wave function in coordinate space and that of classical $\vec{p}$ is

$$
(\hat{P}-\vec{p}) \psi(\vec{x}, t)=-\iota \hbar \frac{\vec{\nabla} A(\vec{x}, t)}{A(\vec{x}, t)} \psi(\vec{x}, t) .
$$

The prefactor $-\iota \hbar \vec{\nabla} A(\vec{x}, t) / A(\vec{x}, t)$ in front of $\psi(\vec{x}, t)$ is imaginary and on the order of $\hbar$. We interpret it as a nonclassical component of $\hat{P}$ vanishing in the $\hbar \rightarrow 0$ limit, with $\vec{p}$ being its classical component. The nonclassical momentum is proportional to the slope of the amplitude or density and this is the quantity that we will use below to approximate the quantum potential

$$
\vec{r}(\vec{x}, t)=2 \frac{\vec{\nabla} A(\vec{x}, t)}{A(\vec{x}, t)}=\frac{\vec{\nabla} \rho(\vec{x}, t)}{\rho(\vec{x}, t)} .
$$

The total energy of a system in this formulation is 


$$
E=\int\left(\frac{\vec{p}^{T} M^{-1} \vec{p}}{2}+V(\vec{x})+U(\vec{x}, t)\right) \rho(\vec{x}, t) d \vec{x} .
$$

The quantum potential $U(\vec{x}, t)$, which is on the order of $\hbar^{2}$, can be considered as a nonclassical contribution to the kinetic energy operator, though it enters equations of motion on equal footing with potential $V(\vec{x})$. Atomic unit of $\hbar=1$ is used below.

Equation (3) is a continuity equation for a normalizable wave function density, which is conserved in a closed system

$$
\lim _{|\vec{x}| \rightarrow \infty} \rho(\vec{x}, t)=0 \quad \text { and } \quad \int \rho(\vec{x}, t) d \vec{x}=1 .
$$

Initial density $\rho(\vec{x}, 0)$ can be discretized in a set of particles moving along trajectories. A certain amount of density within a volume element, $\delta \vec{x}_{i}(t)=\delta x_{i}^{(1)}(t) \delta x_{i}^{(2)}(t) \delta x_{i}^{(3)}(t) \cdot \ldots$, is associated with the $i$ th trajectory. This quantity-the weight of a trajectory $w_{i}$-remains constant in the course of dynamics $^{27}$

$$
\frac{d}{d t}\left(\rho\left(\vec{x}_{i}, t\right) \delta \vec{x}_{i}(t)\right)=0 \quad \text { or } \quad \rho\left(\vec{x}_{i}, t\right) \delta \vec{x}_{i}(t)=w_{i} .
$$

We can use this expression to establish an important property of the quantum potential for a closed system following from the conservation of the total energy and equations of motion:

$$
\frac{d E}{d t}=\int \frac{\partial U(\vec{x}, t)}{\partial t} \rho(\vec{x}, t) d \vec{x}=0 .
$$

Another property of the quantum potential following from its definition (4) is that it exerts zero average force

$$
F^{(n)}=-\int \frac{\partial U(\vec{x}, t)}{\partial x^{(n)}} \rho(\vec{x}, t) d \vec{x}=0 .
$$

These two general properties, Eqs. (10) and (11), can be used to assess the quality of semiclassical methods based on quantum trajectories.

\section{B. Approximating the nonclassical momentum}

As follows from Eq. (4), the quantum potential does not depend on the amplitude of a wave function, only on the curvature of the amplitude. Local interpolation of $\rho(\vec{x}, t)$, evaluation of derivatives of $\rho(\vec{x}, t)$ and subsequent determination of the quantum potential and quantum force become expensive and inaccurate in the region of low density. Therefore, we will look for the optimal approximation to the logderivative of the density, $\vec{r}(\vec{x}, t)$ of Eq. (6), rather than to $\rho(\vec{x}, t)$. Each spacial component of $\vec{r}(\vec{x}, t)$,

$$
r^{(n)}(\vec{x}, t)=\frac{1}{\rho(\vec{x}, t)} \frac{\partial \rho(\vec{x}, t)}{\partial x^{(n)}},
$$

is approximated by a function of $\vec{x}$ of general form, $g^{(n)}(\vec{x}, \vec{s})$, with time-dependent parameters $\vec{s}$. Vector $\vec{s}$ is a combined list of parameters of all $g^{(n)} s$. In general, the dimensionality $K$ of $\vec{s}$ is not directly related to the dimensionality $N$ of $\vec{x}$. For each dimension we define a functional, which after differentiation by parts becomes

$$
\begin{aligned}
I^{(n)} & =\int\left(r^{(n)}(\vec{x}, t)-g^{(n)}(\vec{x}, \vec{s})\right)^{2} \rho(\vec{x}, t) d \vec{x} \\
& =I_{0}^{(n)}+\int\left(2 \frac{\partial g^{(n)}(\vec{x}, \vec{s})}{\partial x^{(n)}}+g^{(n)}(\vec{x}, \vec{s})^{2}\right) \rho(\vec{x}, t) d \vec{x} .
\end{aligned}
$$

$I_{0}^{(n)}$ abbreviates a term that does not depend on $\vec{s}$. After trajectory-discretization of the initial density $\rho(\vec{x}, 0), I^{(n)}$ is expressed as a weighted sum over trajectories

$$
I^{(n)}=I_{0}^{(n)}+\sum_{i} w_{i}\left(2 \frac{\partial g^{(n)}\left(\vec{x}_{i}, \vec{s}\right)}{\partial x^{(n)}}+g^{(n)}\left(\vec{x}_{i}, \vec{s}\right)^{2}\right) .
$$

The fact that neither $\rho(\vec{x}, t)$ nor its derivatives are involved is of crucial importance for efficient global implementation, which in this case will be linear with respect to the number of trajectories.

The optimal values of $\vec{s}$ are found from minimization of the combined functional $I$

$$
I=\sum_{n=1}^{N} \frac{I^{(n)}}{m^{(n)}},
$$

by solving a system of $K$ equations

$$
\frac{\partial I}{\partial s^{(k)}}=0 \quad \text { for } \quad k=1 \ldots K .
$$

The mass factors were introduced in Eq. (15) in order to relate $I$ to the corresponding quantum potential. Once Eqs. (16) are solved, an approximate quantum potential $\widetilde{U}$, and its average $\langle\widetilde{U}\rangle$ can be expressed in terms of $g^{(n)}(\vec{x}, \vec{s})$ using

$$
\begin{aligned}
& \frac{1}{\rho(\vec{x}, t)} \frac{\partial \rho(\vec{x}, t)}{\partial x^{(n)}} \approx g^{(n)}(\vec{x}, \vec{s}) \quad \text { and } \\
& \frac{1}{\rho(\vec{x}, t)} \frac{\partial^{2} \rho(\vec{x}, t)}{\partial x^{(n) 2}} \approx \frac{\partial g^{(n)}(\vec{x}, \vec{s})}{\partial x^{(n)}}+g^{(n)}(\vec{x}, \vec{s})^{2},
\end{aligned}
$$

as

$$
\begin{aligned}
& \widetilde{U}=-\sum_{n=1}^{N} \frac{1}{8 m^{(n)}}\left(g^{(n)}(\vec{x}, \vec{s})^{2}+2 \frac{\partial g^{(n)}(\vec{x}, \vec{s})}{\partial x^{(n)}}\right) \\
& \text { and }\langle\widetilde{U}\rangle=-\frac{1}{8}\left(I-\sum \frac{I_{0}^{(n)}}{m^{(n)}}\right) .
\end{aligned}
$$

For approximate quantum potential evaluated at the optimal values of $\vec{s}$, the condition of the energy conservation, Eq. (10), is satisfied as can be verified using Eqs. (9) and (16)

$$
\begin{aligned}
\frac{d E}{d t} & =\sum_{k=1}^{K} \int \frac{\partial \widetilde{U}}{\partial s^{(k)}} \rho(\vec{x}, t) d \vec{x} \frac{d s^{(k)}}{d t}=-\frac{1}{8} \sum_{k=1}^{K} \frac{\partial I}{\partial s^{(k)}} \frac{d s^{(k)}}{d t} \\
& =0 .
\end{aligned}
$$

\section{Linearized quantum force approximation}

A solution of Eq. (16) will generate the energy conserving dynamics of a closed system with any functional form of $g^{(n)}$. Naturally, one is interested only in physically meaningful and practical forms for $g^{(n)}$. A simple functional form of $g^{(n)}$ yielding analytical solutions of Eq. (16) is highly desirable, but it is $g^{(n)}$ represented in terms of a complete (or a 
sufficiently large) basis, that will lead to accurate quantum dynamics for any system. Considering that we target quantum-mechanical effects in semiclassical systems and that the most successful semiclassical methods give exact time-evolution of a Gaussian wave function in a quadratic potential, we start by assuming that our $g^{(n)}$ s are generated from a Gaussian density

$$
\rho(\vec{x}, t) \approx\left(\frac{\operatorname{det}(A)}{\pi}\right)^{N / 2} \exp \left(-\left(\vec{x}-\vec{x}_{0}\right)^{T} A\left(\vec{x}-\vec{x}_{0}\right)\right) .
$$

$A$ is a symmetric matrix of the widths parameters, and vector $\vec{x}_{0}$ is the center of the Gaussian density. The corresponding $g$ is a linear function of $\vec{x}$. Note here that we do not restrict the actual density to be a Gaussian. We simply look for the best linear approximation of $\vec{r}(\vec{x}, t)$ as outlined in Sec. II B, that is consistent with a Gaussian density and results in a linearized quantum force (LQF). If Eq. (22) becomes a poor approximation to the actual density, then the matrix elements of $A$ and the resulting $\widetilde{U}$ go to zero and dynamics becomes purely classical. With $g^{(n)}$ s derived from Eq. (22), $\vec{g}(\vec{x}, \vec{s})=2 A(\vec{x}$ $-\vec{x}_{0}$ ), the functional (15) becomes

$$
\begin{aligned}
I(\vec{s})= & \int\left(\left(\vec{x}-\vec{x}_{0}\right)^{T} A^{T} M^{-1} A\left(\vec{x}-\vec{x}_{0}\right)\right. \\
& \left.-\operatorname{Tr}\left(A M^{-1}\right)\right) \rho(\vec{x}, t) d \vec{x}+I_{0} .
\end{aligned}
$$

The last term, $I_{0}$, is independent on parameters $\vec{s}$. The list of parameters $\vec{s}$ consists of $N(N+1) / 2$ elements of $A$ and of $N$ components of $\vec{x}_{0}$. Minimization of this functional gives a unique solution which for a normalized density is

$$
\vec{x}_{0}=\langle x\rangle=\int \vec{x} \rho(\vec{x}, t) d \vec{x},
$$

and introducing matrix $B$ of the second moments $b_{i j}$ $=\int\left(x^{(i)}-x_{0}^{(i)}\right)\left(x^{(j)}-x_{0}^{(j)}\right) \rho(\vec{x}, t) d \vec{x}$

$$
A=\frac{1}{2} B^{-1} \text {. }
$$

The corresponding approximate quantum potential and its force have several attractive features. From the point of view of implementation:

(i) Optimization has a simple analytical solution;

(ii) the first and second moments of the density distribution are expressed as single sums over trajectories, $\vec{x}_{0}=\sum_{i} w_{i} \vec{x}$ and $b_{i j}=\sum_{i} w_{i}\left(x^{(i)}-x_{0}^{(i)}\right)\left(x^{(j)}-x_{0}^{(j)}\right)$;

(iii) since our approach is global, the determination of these two moments is the only addition to the classical trajectory propagation at each time step;

(iv) the propagation method is linear with the number of dimensions.

From the theoretical point of view:

(i) Time-evolution of a Gaussian wavepacket in a locally quadratic potential is solved exactly (including correlated density);

(ii) the total energy is conserved;

(iii) the average quantum force is zero: Using Eqs. (19) and (24), the average linearized quantum force is $\vec{F}$ $=A M^{-1} A\left\langle\vec{x}-\vec{x}_{0}\right\rangle=0$; (iv) since $\widetilde{U}$ is based on approximation to the nonclassical component of the momentum operator, which is small in the semiclassical limit, we expect that the LQF method can adequately describe dominant quantum-mechanical effects in semiclassical systems.

\section{The wavepacket analysis}

So far we have formulated our approach in terms of trajectories with their weights determined by the initial density. In order to propagate quantum trajectories in the presence of the approximate quantum potential, we do not need the densities or wave functions explicitly except at $t=0$. Computation of $\widetilde{U}$ is formulated using just the trajectory weights, Eq. (9). The weights and trajectory positions are also sufficient to compute expectation values of the coordinate-dependent operators. For example, an expression for the reaction probability of a wavepacket is

$$
P(t)=\int \hat{P} \rho(\vec{x}, t) d \vec{x} \approx \sum_{i}^{\text {prod }} w_{i} .
$$

Operator $\hat{P}$ projects the density onto products and can be represented as a Heaviside function in reaction coordinate. The summation goes over trajectories, which are located in the product region of a potential surface at time $t$. Other quantities used to define LQF are the moments of the trajectory distribution. Using the hermiticity of the Hamiltonian, we can also directly compute a less trivial phase-sensitive quantity-the time-dependent auto-correlation function of a real wavepacket, which is the central object for calculating photodissociation spectra

$$
\begin{aligned}
C(t) & =\langle\psi(\vec{x}, 0) \mid \psi(\vec{x}, t)\rangle=\left\langle\psi^{\star}(\vec{x}, t / 2) \mid \psi(\vec{x}, t / 2)\right\rangle \\
& =\sum_{i} w_{i} \exp \left(2 \iota S\left(\vec{x}_{i}, t / 2\right)\right) .
\end{aligned}
$$

For analysis of arbitrary wavepackets we need a more general type of integrals - a correlation function of stationary, $\chi(\vec{x}, 0)$, and evolving wavepackets. The correlation function

$$
\begin{aligned}
C(t) & =\int \chi^{\star}(\vec{x}, 0) \psi(\vec{x}, t) d \vec{x} \\
& =\int \chi^{\star}(\vec{x}, 0) \exp (\iota S(\vec{x}, t)) \sqrt{\rho(\vec{x}, t)} d \vec{x},
\end{aligned}
$$

requires knowledge of either the volume element $d \vec{x}$ or of $\rho(\vec{x}, t)$. In one-dimensional applications ${ }^{26}$ we found $d x_{i}$ from adjacent trajectories, which would be cumbersome to implement in many dimensions. Therefore, we resort to approximation of $\rho(\vec{x}, t)$ and possibly of $S(\vec{x}, t)$ or $\psi(\vec{x}, t)$ in a localized region of space, where $|\chi(\vec{x}, 0)|$ evaluated at positions of trajectories at time $t$ is appreciable, $|\chi(\vec{x}, 0)|>\delta$. Several strategies are available. One can approximate the density with a trial function on a subspace of a given "window" function $h(\vec{x}), \rho(\vec{x}, t) \approx G(\vec{a}, \vec{x})$, by minimizing 


$$
F=\int(\rho(\vec{x}, t)-G(\vec{a}, \vec{x}, t))^{2} h(\vec{x}) d \vec{x},
$$

with respect to parameters $\vec{a}$. Computationally, the most straightforward choice is $h(\vec{x})=|\chi(\vec{x}, 0)|$ in conjunction with a polynomial form of $G$ in $\vec{x}$, which will result in a single (for a given time) fitting solved by a system of linear equations for $\vec{a}$. The correlation function in terms of trajectories is

$$
C(t) \approx \sum_{j} \chi^{\star}\left(\vec{x}_{j}, 0\right) \exp \left(\iota S_{j}\right) w_{j} / \sqrt{G\left(\vec{a}, \vec{x}_{j}\right)}
$$

We used this expression with $G$ as the second order polynomial in the numerical examples.

A more controllable, but more expensive option is to approximate $\rho(\vec{x}, t)$ individually for each trajectory, using a narrow window function centered at a trajectory. This procedure scales as the number of trajectories squared, but only a subset of contributing trajectories, satisfying $\left|\chi\left(\vec{x}_{i}, 0\right)\right|>\delta$, need to be considered. Similar to the convolution procedure of Ref. 26, for $j$ th trajectory we chose $h_{j}(\vec{x})=\exp (-(\vec{x}$ $\left.\left.-\vec{x}_{j}\right)^{T} \beta\left(\vec{x}-\vec{x}_{j}\right)\right) . G(\vec{a}, \vec{x})$ was chosen to be either a constant or a quadratic form in $\left(\vec{x}-\vec{x}_{j}\right)$. The widths parameter $\beta$ can be a number or a matrix related to the localization of $|\chi(\vec{x}, 0)|$. $C(t)$ with several values of $\beta$ can be computed simultaneously in order to monitor convergence with respect to the window size. $C(t)$ is found from Eq. (30) with the exception that $G\left(\vec{x}_{j}\right)=a_{j}^{(0)}$ is the zero-order coefficient of the polynomial expansion for each trajectory. We used this formulation for comparison to Eq. (27) in the ICN example.

Yet another possibility is to approximate $\chi^{\star}(\vec{x}, 0)^{2} \psi(\vec{x}, t)^{2} \approx G(\vec{a}, \vec{x})$, and to find complex integrand of $C(t), \chi^{\star}(\vec{x}, 0) \psi(\vec{x}, t)$, directly. The importance of approximating product of squares of the wavepackets is that once again we can obtain equations on $\vec{a}$ in terms of trajectory weights. For a Gaussian $G$ with complex parameters, equations on $\vec{a}$ can be solved analytically by equating the lowest moments, and the resulting $\sqrt{G(\vec{a}, \vec{x})}$ can be integrated analytically to obtain $C(t)$. The square root of a complex number should be taken such that the real and imaginary parts of $C(t)$ are continuous functions of time. As a caution, we note that a more sophisticated trial forms of $G$, that might be desired to check the adequacy of the Gaussian $G$, will result in more complicated equations for $\vec{a}$ that may not have analytical solutions.

All of the methods mentioned above can be used to find the wave function at a given point, if the stationary wavepacket is chosen to be a limiting form of the Dirac $\delta$-function centered around this point. However, we will try to work with correlation functions, rather than wave functions themselves, because the storage and analysis of correlation functions are cheaper. Most quantities in chemical dynamics (energy-resolved reaction probabilities, etc.) can be found by analyzing correlation functions. Obtaining averaged quantities is often less demanding numerically and, for approximate methods, more accurate. In case of the quantum trajectory approach, calculation of a correlation function on a restricted subspace is significantly cheaper.

\section{EXAMPLES AND DISCUSSION}

Our first example illustrates the LQF method in one dimension. We apply it to describe the wavepacket dynamics for the Eckart barrier emphasizing the difference of this method with the approximate quantum potential method, based on the global density fitting. ${ }^{27}$ As a multidimensional illustration we apply the LQF approximation to a benchmark test for nuclear dynamics-calculation of the reaction probability for collinear $\mathrm{H}_{3}$ system, and to photodissociation of a collinear ICN, which is also a standard model problem for studies of approximate propagation methods. The purpose of our testing is twofold: To check the numerical efficiency of LQF and to probe the quality of description it provides.

\section{A. The Eckart barrier}

We consider scattering of a wavepacket on the Eckart barrier, $V=D \cosh ^{-2}(Z x)$, which serves as a simple onedimensional model for the hydrogen exchange reaction. In mass-scaled units the Hamiltonian is $\hat{H}=\hat{p}^{2} / 2+V$. The values of the parameters of $V$ are $D=16$ and $Z=1.3624$. The initial wave function is a Gaussian wavepacket

$$
\psi(0)=\exp \left(-\alpha\left(x-q_{0}\right)^{2}+\iota p_{0}\left(x-q_{0}\right)\right)\left(\frac{2 \alpha}{\pi}\right)^{1 / 4},
$$

located to the left of the barrier in the asymptotic region of $V$ with a positive initial momentum.

Earlier we considered the same problem and obtained energy resolved transmission probabilities using the AQP method, in which quantum potential and quantum force were obtained by fitting the density in terms of a linear combination of Gaussian functions. ${ }^{27}$ It was shown that having full quantum mechanics as the limit of the large number of Gaussian functions and trajectories, the AQP method, in principle, could describe tunneling and interference effects (the latter becoming numerically intensive). It was also shown that the dynamics of the transmitted part of a wavepacket, including the reactant-product wavepacket correlation function and its energy spectrum could be fairly accurately obtained with a single Gaussian density fit. The underlying working equation for obtaining its parameters $\left\{c, a, x_{0}\right\}$,

$$
I^{\mathrm{AQP}}=\int\left(\rho(x)-c^{2} e^{-a^{2}\left(x-x_{0}\right)^{2}}\right)^{2} d x,
$$

was solved by the gradient minimization technique for each time step. The AQP formulation with a single Gaussian density fit looks similar to the LQF method, which is also related to a Gaussian density via Eq. (22). The parameters of Eq. (22) solve a different minimization problem, Eq. (15), and therefore, can be different from their counterparts of Eq. (32). Here we choose parameters of the initial wavepacket $\psi$ that clearly show the difference between the LQF and a single-Gaussian AQP methods.

For the initial parameters of $\psi$ taken as $\alpha=6, q_{0}=-3$ and $p_{0}=4$, the classical initial energy of $\psi$ is half the barrier height. If the quantum potential were set to zero, then all trajectories would remain to the left of the barrier resulting in zero transmission. In the presence of the quantum potential 


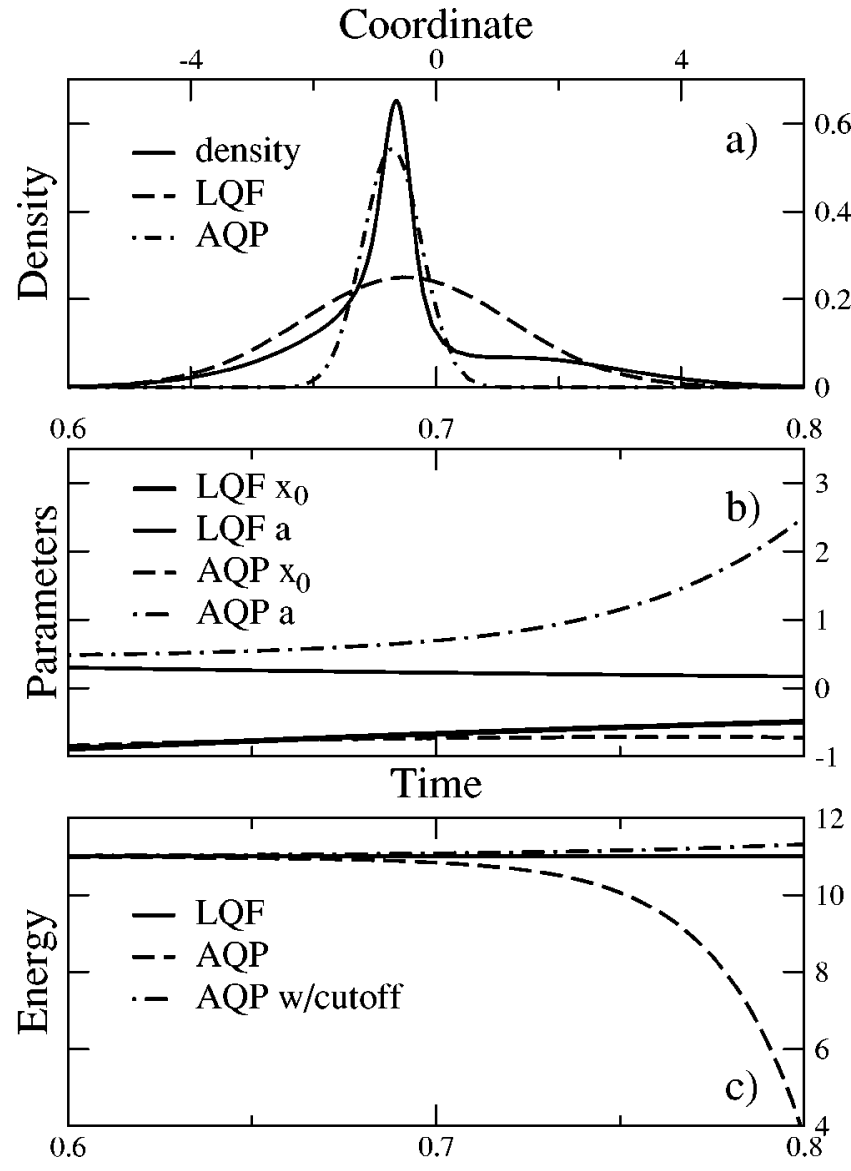

FIG. 1. Dynamics on the Eckart barrier. (a) LQF and AQP approximations of density at $t=0.8$. (b) The width and the position of the center of the fit as functions of time. (c) Total energy of the system as a function of time.

some of the trajectories gain energy sufficient to surmount the barrier, and the wavepacket splits into the reflected and transmitted parts. Let us examine the wavepacket density $\rho$ at a particular instant of time, $t=0.8$, when this splitting occurs. Gaussian For the given choice of initial $\psi$ we obtain qualitatively different approximations of $\rho$. Figure 1(a) shows the density and its approximations obtained with the LQF and AQP methods. The first apparent difference is that while the LQF density covers both reflected and transmitted parts, the AQP density tends to fit the most prominent peak that happens to be to the left of the barrier. This narrow Gaussian severely underestimates density on the tails and leads to unphysically large quantum force resulting in the numerical breakdown of the calculation. This problem could be circumvented by using a convoluted density instead of the optimized density below a certain cutoff of the fitted density, which ensures that quantum force will go to zero at the tails of the distribution. The LQF method is more sensitive to the low-amplitude region: The Gaussian width parameter $2 a$ and the corresponding quantum force go to zero, as the bifurcation of $\psi$ progresses. The AQP width parameter also decreases from 12 to 0.8 as the wavepacket spreads, but after $t=0.7$ it starts to increase, as the density optimization begins to reproduce the dominant peak forming on the left of the barrier. The width parameter and the position of a fitting Gaussian are shown on Fig. 1(b). Positions $x_{0}$ for the two methods begin to differ as $\psi$ bifurcates. The LQF position, $x_{0}=\langle x\rangle$, coincides with the quantum-mechanical expectation of $x$ very well.

The unphysically large quantum force of the AQP method is also manifested in the total energy of the wavepacket, which deviates from its initial value as $\rho$ becomes non-Gaussian in the course of dynamics. The total energy is plotted on Fig. 1(c): The LQF energy is conserved by construction; the AQP energy changes significantly once the unphysically large quantum force is present. Introduction of the cutoff helps in this respect as well as in stabilizing the AQP method. Despite this energy nonconservation, the total amounts of the transmitted density for the two approximate methods are essentially the same (about $10 \%$ less than the quantum-mechanical value), since the wavepacket transmission probability is the half-space averaged and phaseindependent quantity for this simple one-dimensional system. For $\psi$ of initial energy higher than the barrier top ( $p_{0}$ $=6$ as in Ref. 27), optimization procedure of the two methods gave very similar results, including the reactant-product wavepacket correlation functions. In the AQP method the quantum force was approaching zero for long times, and propagation was numerically stable without a cutoff, though the total energy in AQP was not conserved.

The results were obtained using 199 trajectories for both methods. Computationally, the LQF calculation was 15 times faster than the AQP calculation, because the latter involves a nonlinear minimization. The convergence of the LQF parameters with respect to the number of trajectories was better than $10^{-5}$. This suggests, that it might be possible to predetermine parameters for the quantum potential using small number of trajectories and to use these parameters to run large number of trajectories with a sampling adapted to a specific problem.

\section{B. Wavepacket reaction probability for collinear $\mathrm{H}_{3}$}

The collinear hydrogen exchange reaction, $\mathrm{H}_{A}+\mathrm{H}_{B} \mathrm{H}_{C}$ $\rightarrow \mathrm{H}_{A} \mathrm{H}_{B}+\mathrm{H}_{C}$, is a standard test in reaction dynamics. This model system of light nuclei exhibits large quantum effects and provides a fairly stringent test on the quality of approximate dynamical methods. We find the transition probability of a wavepacket for several values of initial kinetic energy for a system of three hydrogen nuclei, as well as for deuterium $\left(\mathrm{D}_{3}\right)$ and tritium $\left(\mathrm{T}_{3}\right)$, which allows one to examine the classical limit, $U=0$, and the isotopic effect on the wavepacket probability. The system is described in the Jacobi coordinates of reactants where the kinetic energy is diagonal. The Hamiltonian, coordinates and potential surface are the same as in Ref. 6.

We start by defining an initial wavepacket

$$
\begin{aligned}
\psi(0)= & \sqrt{\frac{2}{\pi}\left(\alpha_{1} \alpha_{2}\right)^{1 / 2}} \exp \left(-\alpha_{1}\left(R-R_{0}\right)^{2}-\alpha_{2}\left(r-r_{0}\right)^{2}\right. \\
& \left.+\iota p_{0}\left(R-R_{0}\right)\right),
\end{aligned}
$$

where $r$ is the vibrational coordinate of the diatomic, the distance between $\mathrm{H}_{B}$ and $\mathrm{H}_{C}$, and $R$ is the translational degree of freedom-the distance between $\mathrm{H}_{A}$ and the diatomic. $\psi(0)$ is a product of the Gaussian wavepacket in $r$ approxi- 
mating the ground vibrational state with the Gaussian wavepacket in $R$ located in the reactant region and with the momentum directed toward the interaction region. Values of the parameters in atomic units (the unit of time is 918 a.u.) are $R_{0}=4.5, r_{0}=1.3, \alpha_{1}=4, \alpha_{2}=9$ and $p_{0}=[-15,-1]$. The initial positions for quantum trajectories $\vec{x}(0)=\left\{R_{i}, r_{i}\right\}$ are chosen on a rectangular grid with spacings $d R=0.029$ and $d r=0.019$. Trajectory with the small weights $\epsilon<10^{-6}$ are not included. The initial momenta are $\vec{p}(0)=\left\{p_{0}, 0\right\}$ and the initial classical actions are $S_{i}(0)=p_{0}\left(R_{i}-R_{0}\right)$. In the course of dynamics the trajectories arrive either to the product $\left(\mathrm{H}_{A} \mathrm{H}_{B}<\mathrm{H}_{B} \mathrm{H}_{C}\right)$ or back to the reactant region $\left(\mathrm{H}_{A} \mathrm{H}_{B}\right.$ $\left.>\mathrm{H}_{B} \mathrm{H}_{C}\right)$. The wavepacket reaction probability is a value of $P(t)$ of Eq. (26) when the reactant/product separation of the wavepacket is complete and $P(t)$ reaches a plateau. Trajectories were propagated up to time $t_{\max }=1.8$ with the increment $d t=2.5 \times 10^{-3}$. Probabilities were obtained using 3569 trajectories for each value of $p_{0}$. The results are compared with the quantum-mechanical calculations performed with the split-operator method ${ }^{29}$ on a $256 \times 256$ grid and with the classical calculations for which the quantum potential was set identically to zero, $U=0$. The probability calculation were repeated for deuterium and for tritium using 2241 and 1481 trajectories, respectively. The wavepacket parameters for $\mathrm{D}_{3}$ and $\mathrm{T}_{3}$ systems are the same as for $\mathrm{H}_{3}$ with the exception of the range for $p_{0} ; p_{0}=[-2,-22]$ for $\mathrm{D}_{3}$ and $p_{0}$ $=[-3,-27]$ for $T_{3}$.

Some details of the LQF calculation for a single value $p_{0}=-9.5$ for the $\mathrm{H}_{3}$ system are shown on Fig. 2. The wavepacket reaction probability as a function of time, obtained with the LQF method, is similar to the quantum result. The average position in the LQF calculation agrees well with the quantum-mechanical expectation value for short times until the wavepacket "turns around the corner" at $t=0.35$ and splits into transmitted and reflected components. This is also reflected in the LQF width parameters, i.e., in the elements of the matrix $A$ whose elements have their second maximum at about the same time. The off-diagonal element, $a_{R r}$, becomes comparable in magnitude to the diagonal elements showing large amount of correlation between the two coordinates. For later times the elements of $A$ decrease and the quantum force goes to zero because the wave function becomes diffuse in the process of scattering. The behavior of the width parameters suggests that the LQF, due to its simple analytical form, describes the zero-point energy effect for short times when the wave function is mostly localized in the reactant region of the potential, and does not account for it in the product region, something that can be corrected by a more sophisticated trial function $g$. The correct short time description might be still adequate for computation of averaged quantities in semiclassical systems.

Figure 3 shows the wavepacket probabilities as a function of the initial total energy. The classical probabilities are the same for all three isotopes (shown with a single curve) and are quite different from the quantum results. This difference, however, tends to decrease for heavier isotopes, as it should vanish in the infinite mass limit. The agreement between the LQF and quantum probabilities for the three systems is qualitative: The maximal $P(E)$ are overestimated by
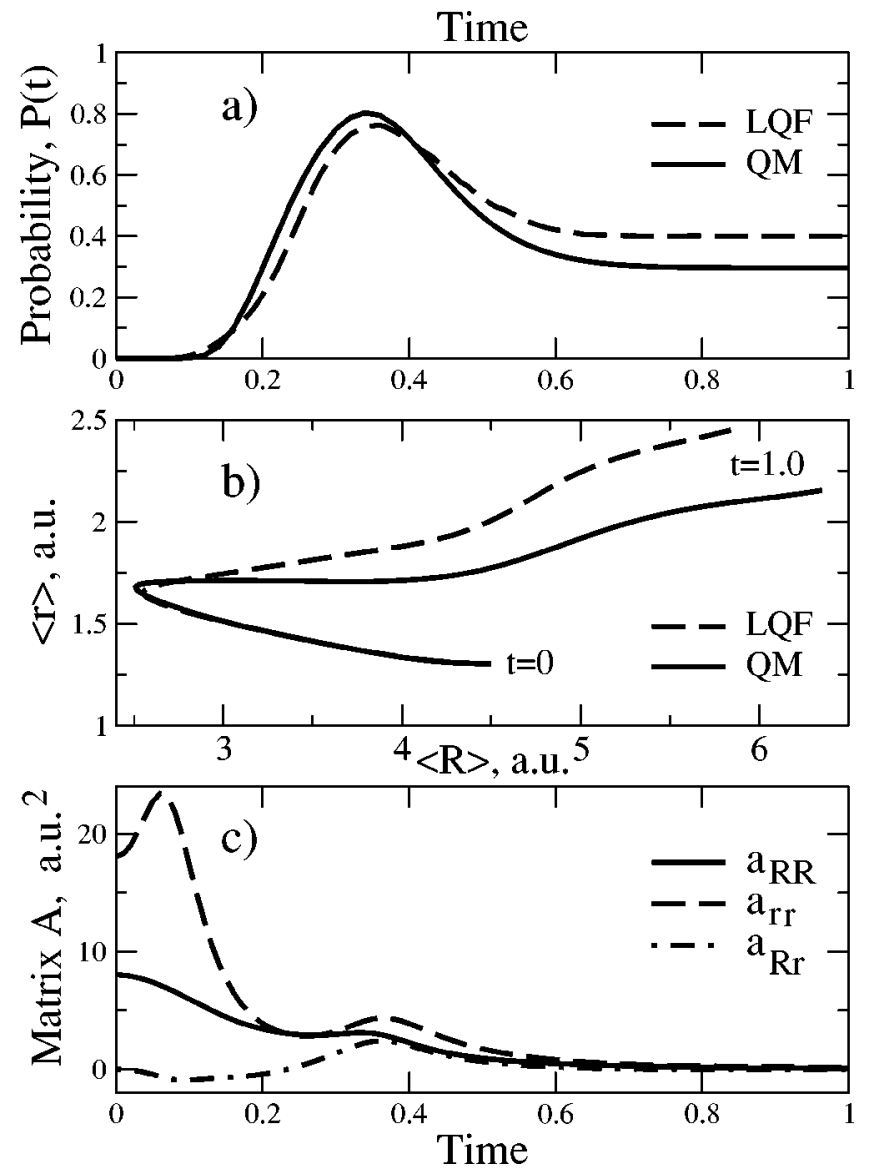

FIG. 2. The collinear hydrogen exchange reaction: (a) The wavepacket reaction probability, $P(t)$, as a function of time; (b) the LQF position parameters and the center of the quantum wavepacket, $\langle R(t)\rangle$ vs $\langle r(t)\rangle$, for times $t=[0.0,1.0]$; (c) the LQF width parameters, i.e., the diagonal matrix elements $a_{R R}$ and $a_{r r}$, and the off-diagonal matrix element $a_{R r}$, as a function of time.

$8-10 \%$ and shifted by about $0.08 \mathrm{eV}$ for all three isotopes, while the overall shape of the LQF curves are similar to the quantum results. The difference between the quantum and LQF probabilities reduces as the quantum potential decreases

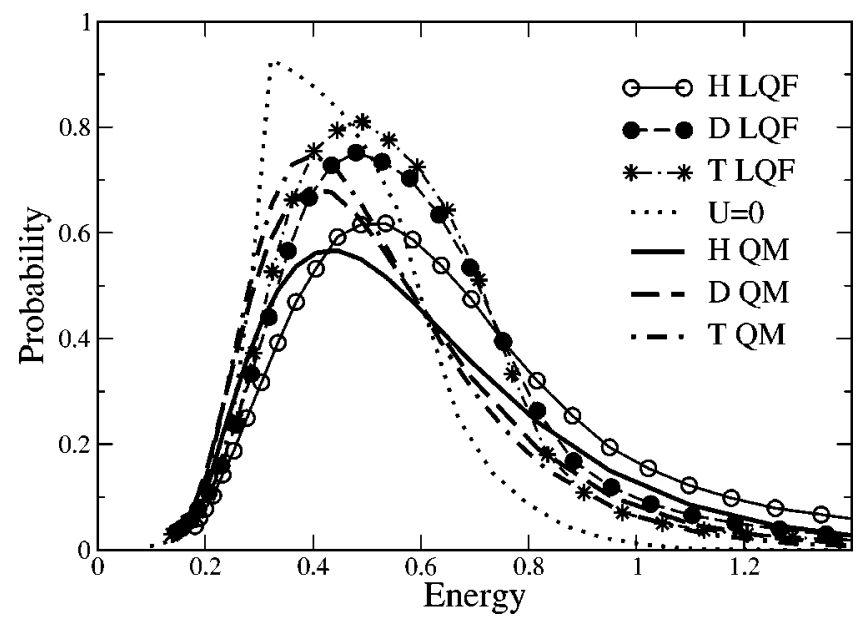

FIG. 3. Transmission $P$ of a wavepacket for a collinear system of three nuclei for $\mathrm{H}_{3}, \mathrm{D}_{3}$, and $\mathrm{T}_{3}$ as a function of initial total energy $E$, obtained with the LQF method (symbols) and quantum mechanically (lines). The dotted line represents quantum trajectory calculations in the absence of the quantum potential, $U=0$, for the three isotopes. 
TABLE I. Parameters of the ground and excited potential surfaces and of the initial wavepacket for ICN in atomic units. $\bar{R}_{\mathrm{CN}}$ and $\bar{R}_{\mathrm{CI}}$ are the equilibrium distances for $\mathrm{CN}$ and CI stretches.

\begin{tabular}{ccccc}
\hline \hline Ground & $k_{\mathrm{CN}}=0.97345 \mathrm{Ha}_{0}^{-2}$ & $\bar{R}_{\mathrm{CN}}=2.1732 a_{0}$ & $k_{\mathrm{Cl}}=0.1863 \mathrm{Ha}_{0}^{-2}$ & $\bar{R}_{\mathrm{CI}}=4.006 \mathrm{a}_{0}$ \\
Excited & $k_{\mathrm{CN}}=0.6576 \mathrm{Ha}_{0}^{-2}$ & $\bar{R}_{\mathrm{CN}}=2.3295 a_{0}$ & $A=7349.9 \mathrm{H}$ & $a=3.535 a_{0}^{-1}$ \\
$\psi(0)$ & $\alpha_{1}=55.1525 \mathrm{a}_{0}^{-2}$ & $\alpha_{2}=42.2053 \mathrm{a}_{0}^{-2}$ & $\alpha_{12}=4.3893 \mathrm{a}_{0}^{-2}$ & $N_{\text {traj }}=167$ \\
\hline \hline
\end{tabular}

relative to the kinetic energy, i.e., for larger mass and higher initial total energy. The discrepancy for the energies close to the top of the barrier (around $0.4 \mathrm{eV}$ ) persists for all masses, which can be explained as follows. In order to have the same $U=0$ limit we used identical wavepackets for all three isotopes, while the ground state depends on the mass. Compared to the ground state, our initial wavepackets were wider and the quadratic approximation to the potential was less accurate for heavier isotopes.

Thus far we conclude that LQF may be suitable to analyze isotope effects in semiclassical molecular systems. For more detailed information, such as the energy-resolved reaction probability, one should be able to account for the zeropoint energy effects on the product side of the potential. Choice of the coordinate system should also play a role on the LQF description of the zero-point energy effect. For example, using the Cartesian coordinates for this problem would be of great interest. Also having a criterion on the applicability of semiclassical methods would be highly useful for the development of a rigorous semiclassical propagation method. For the quantum trajectory method with approximate quantum potential, a cumulative over time global quantity, which accounts for local nonharmonicity, might serve this purpose. Such criterion may be applicable to a wide range of semiclassical methods.

\section{Photodissociation cross section of ICN}

In the final example - the photodissociation of ICN-we compute a phase-dependent quantity in two different ways: With and without approximation to the time-dependent density. We follow works of Heller ${ }^{30}$ and Coalson and Karplus, ${ }^{31}$ where collinear ICN was treated within the Beswick-Jortner model ${ }^{32} \mathrm{~A}$ wavepacket representing an ICN molecule is excited by a laser from the ground to the excited electronic state, where it dissociates into I and CN. The Hamiltonian and the Jacobi coordinate system are described in Ref. 31 . An initial wave function $\psi(0)=\left(2 \sqrt{\alpha_{1} \alpha_{2}} / \pi\right)^{1 / 2} \exp \left(-\alpha_{1}(y\right.$ $\left.\left.-y_{0}\right)^{2}-\alpha_{2}\left(x-x_{0}\right)^{2}+2 \alpha_{12}\left(y-y_{0}\right)\left(x-x_{0}\right)\right)$ is defined as the lowest eigenstate of the ground electronic surface with zero momentum. The ground state potential is composed of two harmonic potentials in $\mathrm{CN}$ and $\mathrm{CI}$ stretches. Thus, $\psi(0)$ is a correlated Gaussian wavepacket in the Jacobi coordinates located on the repulsive wall of the excited surface. The photodissociation cross section is computed from the Fourier transform of the wavepacket auto-correlation function $C(t)$ $=\langle\psi(0) \mid \psi(t)\rangle$

$$
\sigma(\omega)=\omega \Re\left(\int C(t) \exp (\iota \omega t) d t\right) .
$$

The physical value of the repulsion parameter of the excited potential surface yields a rather simple dissociation dy- namics. $C(t)$ decays on the time scale of about one and a half oscillations of the $\mathrm{CN}$ stretch. The LQF spectrum agrees with the quantum result very well as was reported in Ref. 28. The second value of the repulsion parameter, which is three times larger than its physical value, yields a predissociation process (system II in Ref. 31). Numerical values of the parameters of the surfaces and $\psi(0)$ are organized in Table I and presented in atomic units. Trajectories sampling $|\psi(0)|^{2}$ are initialized on a rectangular grid with zero initial momenta and phase. A total of 167 trajectories whose weights exceed $\delta=10^{-4}$ are propagated with a time step $d t=1.25$ a.u. for up to 2500 a.u. The LQF parameters are shown on Fig. 4. Figure 4(a) shows the average position of the wavepacket and initial positions of some of the trajectories sampling $|\psi(0)|^{2}$; the average position, plotted as $\langle y\rangle$ vs $\langle x\rangle$, illustrates how the distance between I and center of mass of $\mathrm{CN}$ increases in the course of dissociation as the $\mathrm{CN}$ stretch undergoes three vibrations. Figure 4(b) shows the matrix elements of $A: a_{11}$ represents the changing width of the wave-
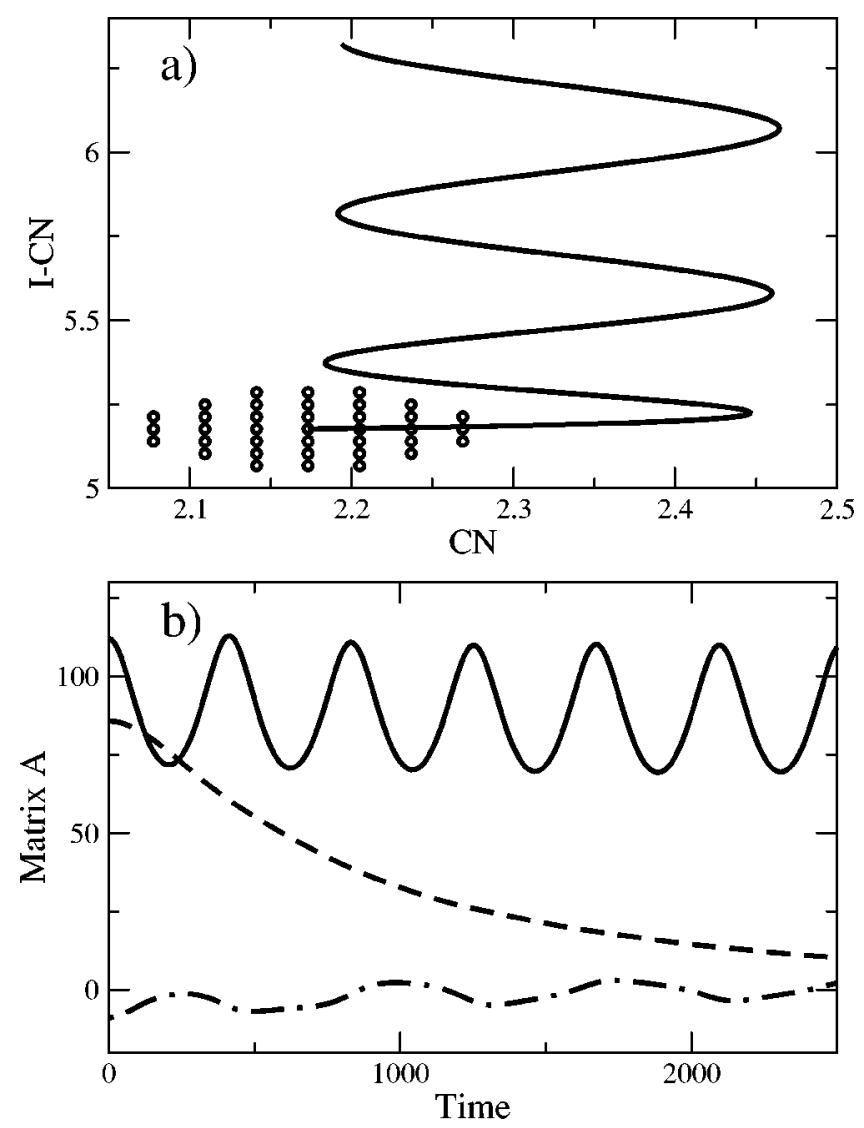

FIG. 4. LQF parameters for ICN: (a) The average position, $\langle y\rangle$ vs $\langle x\rangle$, of the wavepacket and initial positions of trajectories; (b) width parameters $a_{11}$ (solid line), $a_{22}$ (dashed line), and $a_{12}$ (dot-dashed line). 
packet in $\mathrm{CN}$ mode, $a_{22}$ describes the spreading in the dissociation mode. $a_{12}$, which is one-half of the coupling term, also shows oscillations and changes sign in the course of dynamics.

Working with a real $\psi(0)$ has the advantage for the LQF formulation that its auto-correlation function can be easily found by simply summing over the trajectory weights with the phase factors, as given by Eq. (27). In this expression $C(t)$ depends on the quality of the wave function at half-time $t / 2$ on the whole space. We can compare accuracy of the LQF method to a situation when the trajectories are propagated for the whole time $t$, but $C(t)$ is obtained from a localized region of space where $\psi(0)$ is appreciable using Eq. (30). The LQF method is exact for a Gaussian wavepacket in the presence of a locally quadratic potential. For a general potential, the quality of the LQF description will deteriorate as $\psi$ becomes non-Gaussian as time increases and as the wave function becomes diffuse. Comparison of the two correlation functions shows the interplay of shorter time dynamics using Eq. (27) with the analysis of a wavepacket within a localized window using Eq. (30).

Applicability of Eq. (30) for $C(t)$ clearly depends on the spacial extent of the features of $\psi(t)$ compared to the window function: They have to be wide for a polynomial to be accurate. Therefore, for an auto-correlation function we cannot use a single window function; we perform a local expansion around each trajectory as outlined in Sec. II D. The width matrix of the window function is a diagonal matrix with the elements $\left\{16 a_{11}, 16 a_{22}\right\}$. The two correlation functions are shown on Fig. 5 along with the corresponding spectra and the quantum result. One can see that two approximate correlation functions are slightly different from each other. This can be understood as follows: During approximate dynamics accuracy of LQF decreases with time as the wavepacket looses its Gaussian shape and the error in the resulting wave function is not uniform in space. From Fig. 5(a) we see that Eq. (27) describes more accurately the first recurrence, since it comes from half the propagation time. However, its agreement deteriorates exaggerating the next peak as $\psi(t)$ becomes more delocalized for longer times. Equation (30) has contributions only from a region of space with nonzero initial density and does not have this feature. The corresponding spectra are shown on Fig. 5(b). Both spectra agree quite well with the quantum-mechanical result, with results obtained from Eq. (30) being slightly more accurate.

\section{CONCLUSIONS}

We have described a general approach to construction of the energy-conserving approximations to the quantum potential, in which it is determined from the optimal fit of the non-classical component of the momentum operator, restricted by a trial function. As its simplest implementation we used a linear trial function for the nonclassical momentum in many dimensions, that produces linearized quantum force (LQF) and can be related to an approximation of density in terms of a single correlated Gaussian. The equations for optimal parameters are solved analytically in terms of the first and second moments of the trajectory distribution. Formulation in terms of summation over the trajectory distribu-
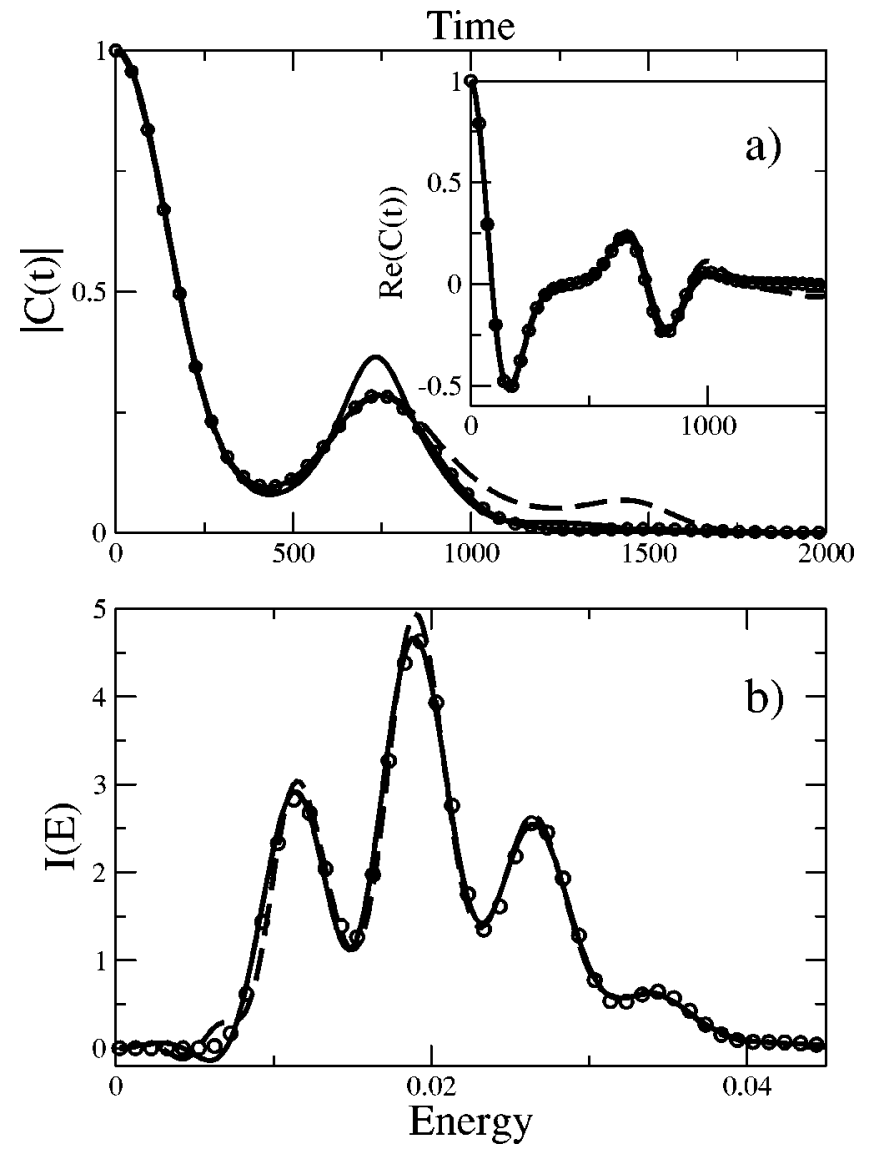

FIG. 5. Photodissociation cross section of ICN: (a) Absolute value of the auto-correlation function (real part on the inset); (b) corresponding spectra. LQF results are shown with solid line when using Eq. (27) and with a dash when using Eq. (30). Quantum results are marked with circles.

tion gives a propagation scheme which is linear with respect to the number of trajectories. Approximating the logderivative of the density eliminates numerical instabilities associated with low-density regions. LQF is exact for a Gaussian wavepacket in a locally quadratic potential, and in addition to the energy conservation satisfies a criterion for the average quantum force to be zero. LQF can be viewed as a simple model of quantum dynamics. Its implementation is computationally cheap and approaches pure classical trajectory propagation.

Our numerical applications to the collinear hydrogen exchange reaction and to the photodissociation of ICN suggest that LQF can accurately describe dominant quantum effects in semiclassical systems. Future multidimensional applications will show if $\mathrm{LQF}$ is a viable model for molecular dynamics. More sophisticated trial functions are also of great interest, since they will provide a systematic way of going from classical to exact quantum dynamics. Development of a criterion on the accuracy of semiclassical methods is highly desirable and it is a subject of our research.

\section{ACKNOWLEDGMENTS}

Acknowledgment is made to the Donors of the American Chemical Society Petroleum Research Fund, for support of this research. This work was also supported by the NSF/ EPSCOR under Grant No. EPS-0296165. 
${ }^{1}$ J. C. Light and T. Carrington, Jr., Adv. Chem. Phys. 114, 263 (2000).

${ }^{2}$ M. Herman and E. Kluk, Chem. Phys. 91, 27 (1984).

${ }^{3}$ K. G. Kay, J. Chem. Phys. 100, 4377 (1994).

${ }^{4}$ W. H. Miller, J. Phys. Chem. A 105, 2942 (2001).

${ }^{5}$ M. L. Brewer, J. S. Hulme, and D. E. Manolopoulos, J. Chem. Phys. 106, 4832 (1997)

${ }^{6}$ S. Garashchuk and D. J. Tannor, Chem. Phys. Lett. 262, 477 (1996).

${ }^{7}$ H. B. W. X. Sun and W. H. Miller, J. Chem. Phys. 109, 7064 (1998).

${ }^{8}$ O. Kuhn and N. Makri, J. Phys. Chem. A 103, 9487 (1999).

${ }^{9}$ S. M. Anderson, J. Ka, P. M. Felker, and D. Neuhauser, Chem. Phys. Lett. 328, 516 (2000).

${ }^{10}$ V. Guallar, V. S. Batista, and W. H. Miller, J. Chem. Phys. 110, 9922 (1999).

${ }^{11}$ V. Guallar, B. F. Gherman, and W. H. Miller, J. Am. Chem. Soc. 124, 3377 (2002).

${ }^{12}$ V. Guallar, V. S. Batista, and W. H. Miller, J. Chem. Phys. 113, 9510 (2000).

${ }^{13}$ K. G. Kay, J. Chem. Phys. 101, 2250 (1994).

${ }^{14}$ A. Walton and D. Manolopoulos, Chem. Phys. Lett. 244, 448 (1995).

${ }^{15}$ D. Bohm, Phys. Rev. 85, 167 (1952).

${ }^{16}$ C. L. Lopreore and R. E. Wyatt, Phys. Rev. Lett. 82, 5190 (1999).
${ }^{17}$ R. E. Wyatt and E. R. Bittner, J. Chem. Phys. 113, 8898 (2000).

${ }^{18}$ D. Nerukh and J. H. Frederick, Chem. Phys. Lett. 332, 145 (2000).

${ }^{19}$ B. K. Dey, A. Askar, and H. Rabitz, J. Chem. Phys. 109, 8770 (1998).

${ }^{20}$ A. Donoso and C. C. Martens, Phys. Rev. Lett. 87, 223202 (2001).

${ }^{21}$ E. R. Bittner, J. Chem. Phys. 115, 6309 (2002).

${ }^{22}$ R. E. Wyatt, C. L. Lopreore, and G. Parlant, J. Chem. Phys. 114, 5113 (2001).

${ }^{23}$ R. E. Wyatt and K. Na, Phys. Rev. E 65, 016702 (2002).

${ }^{24}$ P. R. Holland, The Quantum Theory of Motion (Cambridge University Press, Cambridge, 1993).

${ }^{25}$ A. S. Sanz, F. Borondo, and S. Miret-Artes, J. Phys. 14, 6109 (2002).

${ }^{26}$ S. Garashchuk and V. A. Rassolov, Chem. Phys. Lett. 364, 562 (2002).

${ }^{27}$ S. Garashchuk and V. A. Rassolov, J. Chem. Phys. 118, 2482 (2003).

${ }^{28}$ S. Garashchuk and V. A. Rassolov, Chem. Phys. Lett. 376, 358 (2003).

${ }^{29}$ M. D. Feit, J. A. Fleck, and A. Steiger, J. Comput. Phys. 47, 412 (1982).

${ }^{30}$ R. C. Brown and E. J. Heller, J. Chem. Phys. 75, 186 (1981).

${ }^{31}$ R. D. Coalson and M. Karplus, J. Chem. Phys. 93, 3919 (1990).

${ }^{32}$ J. A. Beswick and J. Jortner, Chem. Phys. 24, 1 (1977).

${ }^{33}$ Single superscript indexes are used to label dimensions; subscript indexes are used to label trajectories. 